Bull. Korean Math. Soc. 49 (2012), No. 3, pp. 445-454

http://dx.doi.org/10.4134/BKMS.2012.49.3.445

\title{
TRANSVERSE KILLING FORMS ON A KÄHLER FOLIATION
}

\author{
Seoung Dal Jung and Min Joo Jung
}

AbStract. On a closed, connected Riemannian manifold with a Kähler foliation $\mathcal{F}$ of codimension $q$, any transverse Killing $r$-form $(2 \leq r \leq q)$ is parallel.

\section{Introduction}

On a Riemannian foliation $\mathcal{F}$ of codimension $q$, transverse conformal Killing forms are defined to be basic forms $\phi$ such that for any vector field $X$ normal to the foliation,

$$
\nabla_{X} \phi=\frac{1}{r+1} i(X) d \phi+\frac{1}{q-r+1} X^{*} \wedge \delta_{T} \phi
$$

where $r$ is the degree of the form $\phi$ and $X^{*}$ the dual 1-form of $X$. See Section 3 for the definition of $\delta_{T}$. The transverse conformal Killing forms with $\delta_{T} \phi=0$ are called transverse Killing forms. Transverse Killing forms (resp. transverse conformal Killing forms) are generalizations of transversal Killing fields (resp. transversal conformal Killing fields) [5]. Many authors have studied the Killing forms and conformal Killing forms on a Riemannian manifold [9, 11, 15, 16, 17]. Recently, Jung and Richardson [5] proved that on a Riemannian foliation with a non-positive curvature endomorphism, any transverse Killing forms on $M$ are parallel. In [5], they assumed that the mean curvature form $\kappa$ satisfies $\delta_{B} \kappa_{B}=0$. In this paper, we prove the result in [5] without the condition $\delta_{B} \kappa_{B}=0$ (Theorem 3.3). Moreover, we prove that on a Kähler foliation on a compact manifold, any transverse Killing $r$-form $(2 \leq r \leq q)$ is parallel (Theorem 3.11). Note that the curvature condition does not need to prove Theorem 3.11.

\section{Preliminaries}

Let $\left(M, g_{M}, \mathcal{F}\right)$ be a $(p+q)$-dimensional Riemannian manifold with a foliation $\mathcal{F}$ of codimension $q$ and a bundle-like metric $g_{M}$ with respect to $\mathcal{F}$. Then

Received March 30, 2010; Revised April 2, 2012.

2010 Mathematics Subject Classification. 53C12, 53C55.

Key words and phrases. transverse Killing form, transverse conformal Killing form, Kähler foliation. 
we have an exact sequence of vector bundles

$$
0 \longrightarrow L \longrightarrow T M \stackrel{\pi}{\longrightarrow} Q \longrightarrow 0,
$$

where $L$ is the tangent bundle and $Q=T M / L$ is the normal bundle of $\mathcal{F}$. The metric $g_{M}$ determines an orthogonal decomposition $T M=L \oplus L^{\perp}$, identifying $Q$ with $L^{\perp}$ and inducing a metric $g_{Q}$ on $Q$. The metric is bundle-like if and only if $\theta(X) g_{Q}=0$ for every $X \in \Gamma L$, where $\theta(X)$ is the transverse Lie derivative [18, 19]. Let $V(\mathcal{F})$ be the space of all vector fields $Y$ on $M$ satisfying $[Y, Z] \in \Gamma L$ for all $Z \in \Gamma L$. An element of $V(\mathcal{F})$ is called an infinitesimal automorphism of $\mathcal{F}[3,13]$. Let

$$
\bar{V}(\mathcal{F})=\{\bar{Y}:=\pi(Y) \mid Y \in V(\mathcal{F})\} .
$$

Then we have an associated exact sequence of Lie algebras

$$
0 \longrightarrow \Gamma L \longrightarrow V(\mathcal{F}) \stackrel{\pi}{\longrightarrow} \bar{V}(\mathcal{F}) \longrightarrow 0 \text {. }
$$

Let $\nabla$ be the transverse Levi-Civita connection on $Q$, which is torsion-free and metric with respect to $g_{Q}[6]$. Let $R^{\nabla}, K^{\nabla}, \rho^{\nabla}$ and $\sigma^{\nabla}$ be the transversal curvature tensor, transversal sectional curvature, transversal Ricci operator and transversal scalar curvature with respect to $\nabla$, respectively. Let $\Omega_{B}^{*}(\mathcal{F})$ be the space of all basic forms on $M$, i.e.,

$$
\Omega_{B}^{*}(\mathcal{F})=\left\{\omega \in \Omega^{*}(M) \mid i(X) \omega=0, i(X) d \omega=0, \quad \forall X \in \Gamma L\right\} .
$$

Then $\Omega^{*}(M)$ is decomposed as [1]

$$
\Omega(M)=\Omega_{B}(\mathcal{F}) \oplus \Omega_{B}(\mathcal{F})^{\perp} .
$$

We have $\Omega_{B}^{r}(\mathcal{F}) \subset \Gamma\left(\Lambda^{r} Q^{*}\right)$ and $\bar{V}(\mathcal{F}) \cong \Omega_{B}^{1}(\mathcal{F})$. Now we define the connection $\nabla$ on $\Omega_{B}^{*}(\mathcal{F})$, which is induced from the connection $\nabla$ on $Q$ and Riemannian connection $\nabla^{M}$ of $g_{M}$. Let $\phi_{B}$ be the basic part of $\phi$. The exterior differential on the de Rham complex $\Omega^{*}(M)$ restricts a differential $d_{B}: \Omega_{B}^{r}(\mathcal{F}) \rightarrow \Omega_{B}^{r+1}(\mathcal{F})$. Let $\kappa \in Q^{*}$ be the mean curvature form of $\mathcal{F}$. Then it is well known that $\kappa_{B}$ is closed [1]. The star operator $\bar{*}: \Omega_{B}^{r}(\mathcal{F}) \rightarrow \Omega_{B}^{r+1}(\mathcal{F})$ is given $[14,18]$ by

$$
\bar{*} \phi=(-1)^{p(q-r)} *\left(\phi \wedge \chi_{\mathcal{F}}\right), \quad \forall \phi \in \Omega_{B}^{r}(\mathcal{F}),
$$

where $\chi_{\mathcal{F}}$ is the characteristic form of $\mathcal{F}$ and $*$ is the Hodge star operator associated to $g_{M}$. Then the pointwise inner product $\langle$,$\rangle on \Lambda^{r} Q^{*}$ is welldefined and the formal adjoint $\delta_{B}: \Omega_{B}^{r}(\mathcal{F}) \rightarrow \Omega_{B}^{r-1}(\mathcal{F})$ of $d_{B}$ is given $[5,14]$ by

$$
\delta_{B} \phi=(-1)^{q(r+1)+1} \bar{*} d_{T} \bar{*} \phi=\delta_{T} \phi+i\left(\kappa_{B}^{\sharp}\right) \phi,
$$

where $(\cdot)^{\sharp}$ is $g_{Q}$-dual vector field to $(\cdot), d_{T}=d-\kappa_{B} \wedge$ and $\delta_{T}=(-1)^{q(r+1)+1} \bar{*} d \bar{*}$ is the formal adjoint operator of $d_{T}$ with respect to $\Omega_{B}^{r}(\mathcal{F})$. The basic Laplacian $\Delta_{B}$ is given by $\Delta_{B}=d_{B} \delta_{B}+\delta_{B} d_{B}$ [14]. Now we recall the generalized maximum principles for later use. 
Lemma 2.1 ([4]). Let $\mathcal{F}$ be a Riemannian foliation on a closed, connected Riemannian manifold $\left(M, g_{M}\right)$. If $\left(\Delta_{B}-\kappa_{B}^{\sharp}\right) f \geq 0($ or $\leq 0)$ for any basic function $f$, then $f$ is constant.

Let $\mathcal{H}_{B}^{r}(\mathcal{F})=\operatorname{Ker} \Delta_{B}$ be the set of the basic-harmonic forms of degree $r$. Then we have $[8,14]$

$$
\Omega_{B}^{r}(\mathcal{F})=\mathcal{H}_{B}^{r}(\mathcal{F}) \oplus \operatorname{im} d_{B} \oplus \operatorname{im} \delta_{B}
$$

with finite dimensional $\mathcal{H}_{B}^{r}(\mathcal{F})$. Let $\left\{E_{a}\right\}(a=1, \ldots, q)$ be a local orthonormal basis of $Q$ such that $\left(\nabla E_{a}\right)_{x}=0$ for $x \in M$ and $\left\{\theta^{a}\right\}$ a $g_{Q^{-d u a l}}$ basic 1 -forms on $Q^{*}$. Let $\nabla_{\mathrm{tr}}^{*}$ be a formal adjoint of $\nabla_{\mathrm{tr}}=\sum_{a=1}^{q} \theta^{a} \otimes \nabla_{E_{a}}: \Omega_{B}^{r}(\mathcal{F}) \rightarrow$ $Q^{*} \otimes \Omega_{B}^{r}(\mathcal{F})$. Then $\nabla_{\mathrm{tr}}^{*}=-\sum_{a=1}^{q}\left(i\left(E_{a}\right) \otimes \mathrm{id}\right) \nabla_{E_{a}}+\left(i\left(\kappa_{B}^{\sharp}\right) \otimes \mathrm{id}\right)$, and so

$$
\nabla_{\mathrm{tr}}^{*} \nabla_{\mathrm{tr}}=-\sum_{a=1}^{q} \nabla_{E_{a}, E_{a}}^{2}+\nabla_{\kappa_{B}^{\sharp}}: \Omega_{B}^{r}(\mathcal{F}) \rightarrow \Omega_{B}^{r}(\mathcal{F}),
$$

where $\nabla_{X, Y}^{2}=\nabla_{X} \nabla_{Y}-\nabla_{\nabla_{X}^{M} Y}$ for any $X, Y \in T M$. The operator $\nabla_{\mathrm{tr}}^{*} \nabla_{\mathrm{tr}}$ is positive definite and formally self adjoint on the space of basic forms [2]. We define the bundle map $A_{Y}: \Lambda^{r} Q^{*} \rightarrow \Lambda^{r} Q^{*}$ for any $Y \in V(\mathcal{F})$ [7] by

$$
A_{Y} \phi=\theta(Y) \phi-\nabla_{Y} \phi
$$

where $\theta(Y)$ is the transverse Lie derivative. Since $\theta(X) \phi=\nabla_{X} \phi$ for any $X \in \Gamma L, A_{Y}$ preserves the basic forms and depends only on $\bar{Y}$. Now, we recall the generalized Weitzenböck formula.

Theorem $2.2([2])$. On a Riemannian foliation $\mathcal{F}$, we have

$$
\Delta_{B} \phi=\nabla_{\mathrm{tr}}^{*} \nabla_{\mathrm{tr}} \phi+F(\phi)+A_{\kappa_{B}^{\sharp}} \phi, \quad \phi \in \Omega_{B}^{r}(\mathcal{F}),
$$

where $F(\phi)=\sum_{a, b=1}^{q} \theta^{a} \wedge i\left(E_{b}\right) R^{\nabla}\left(E_{b}, E_{a}\right) \phi$. If $\phi$ is a basic 1-form, then $F(\phi)^{\sharp}=\rho^{\nabla}\left(\phi^{\sharp}\right)$.

From Theorem 2.2, we have the following. For any $\phi \in \Omega_{B}^{r}(\mathcal{F})$,

$$
\frac{1}{2} \Delta_{B}|\phi|^{2}=\left\langle\Delta_{B} \phi, \phi\right\rangle-\left|\nabla_{\operatorname{tr}} \phi\right|^{2}-\langle F(\phi), \phi\rangle-\left\langle A_{\kappa_{B}^{\sharp}} \phi, \phi\right\rangle .
$$

Then we have the following.

Theorem 2.3 ([10]). Let $\left(M, g_{M}, \mathcal{F}\right)$ be a compact Riemannian manifold with a Riemannian foliation $\mathcal{F}$ and a bundle-like metric $g_{M}$. If $F$ is non-negative and positive at some point, then

$$
\mathcal{H}_{B}^{r}(\mathcal{F})=\{0\} .
$$

If $\rho^{\nabla}$ is non-negative and positive at some point, then

$$
\mathcal{H}_{B}^{1}(\mathcal{F})=\{0\} .
$$




\section{Transverse Killing forms}

Let $\left(M, g_{M}, \mathcal{F}\right)$ be a Riemannian manifold with a Kähler foliation $\mathcal{F}$ of codimension $q=2 m$ and a bundle-like metric $g_{M}$ [12]. Note that for any $X, Y \in \Gamma Q$

$$
\Omega(X, Y)=g_{Q}(X, J Y)
$$

defines a basic 2-form $\Omega$, which is closed as consequence of $\nabla g_{Q}=0$ and $\nabla J=0$, where $J: Q \rightarrow Q$ is an almost complex structure on $Q$. Then we have

$$
\Omega=-\frac{1}{2} \sum_{a=1}^{2 m} \theta^{a} \wedge J \theta^{a} .
$$

Moreover, we have the following identities:

$$
R^{\nabla}(X, Y) J=J R^{\nabla}(X, Y), \quad R^{\nabla}(J X, J Y)=R^{\nabla}(X, Y),
$$

where $X$ and $Y$ are elements of $\Gamma Q$.

Definition 3.1. A basic $r$-form $\phi \in \Omega_{B}^{r}(\mathcal{F})$ is called a transverse conformal Killing $r$-form if for any vector field $X \in \Gamma Q$,

$$
\nabla_{X} \phi=\frac{1}{r+1} i(X) d_{B} \phi-\frac{1}{q-r+1} X^{*} \wedge \delta_{T} \phi
$$

where $\delta_{T}=\delta_{B}-i\left(\kappa_{B}^{\sharp}\right)$. In addition, if the basic $r$-form $\phi$ satisfies $\delta_{T} \phi=0$, it is called a transverse Killing $r$-form.

Note that a transverse conformal Killing 1-form (resp. transverse Killing 1 -form) is a $g_{Q}$-dual form of a transversal conformal Killing vector field (resp. transversal Killing vector field).

Proposition $3.2([5])$. Any basic $r(r \geq 1)$-form $\phi$ is a transverse Killing form if and only if

$$
\Delta_{B} \phi=\frac{r+1}{r} F(\phi)+\theta\left(\kappa_{B}^{\sharp}\right) \phi .
$$

By Lemma 2.1, we have the following (cf. [5]).

Theorem 3.3. Let $\left(M, g_{M}, \mathcal{F}\right)$ be a closed, connected Riemannian manifold with a foliation $\mathcal{F}$ of codimension $q$ and a bundle-like metric $g_{M}$. Assume that $F$ is non-positive. Then any transverse Killing $r(1 \leq r \leq q-1)$-forms are parallel. In addition, if $F$ is negative at some point, then for $1 \leq r \leq q-1$, there are no transverse Killing $r$-forms on $M$.

Proof. Let $\phi$ be a transverse Killing $r$-form. From (2.12) and (3.5), we have

$$
\frac{1}{2}\left(\Delta_{B}-\kappa_{B}^{\sharp}\right)|\phi|^{2}=\frac{1}{r}\langle F(\phi), \phi\rangle-\left|\nabla_{\mathrm{tr}} \phi\right|^{2} .
$$


Hence, if $F$ is non-positive, then $\left(\Delta_{B}-\kappa_{B}^{\sharp}\right)|\phi|^{2} \leq 0$. By Lemma 2.1, $|\phi|$ is constant. Hence we have

$$
\frac{1}{r}\langle F(\phi), \phi\rangle-\left|\nabla_{\mathrm{tr}} \phi\right|^{2}=0
$$

This equation implies that $\phi=0$ under the curvature condition.

Corollary 3.4. Let $\left(M, g_{M}, \mathcal{F}\right)$ be as in Theorem 3.3. Assume the transversal Ricci curvature $\rho^{\nabla}$ is non-positive and negative at some point. Then there are no transversal Killing vector fields on $M$.

Remark. Theorem 3.3 and Corollary 3.4 have been proved in [5] under the condition $\delta_{B} \kappa_{B}=0$.

Lemma 3.5. On a Kähler foliation $\mathcal{F}$ of codimension $q=2 m$, we have

$$
\sum_{a, b=1}^{2 m} i\left(E_{a}\right) i\left(E_{b}\right) R^{\nabla}\left(E_{a}, J E_{b}\right)=0 .
$$

Proof. Since $R^{\nabla}(X, Y)=R^{\nabla}(J X, J Y)$ for any $X, Y \in \Gamma Q$, we get

$$
\begin{aligned}
\sum_{a, b=1}^{2 m} i\left(E_{a}\right) i\left(E_{b}\right) R^{\nabla}\left(E_{b}, J E_{a}\right) & =-\sum_{a, b=1}^{2 m} i\left(E_{a}\right) i\left(E_{b}\right) R^{\nabla}\left(J E_{b}, E_{a}\right) \\
& =\sum_{a, b=1}^{2 m} i\left(E_{a}\right) i\left(E_{b}\right) R^{\nabla}\left(E_{a}, J E_{b}\right) \\
& =-\sum_{a, b=1}^{2 m} i\left(E_{b}\right) i\left(E_{a}\right) R^{\nabla}\left(E_{a}, J E_{b}\right),
\end{aligned}
$$

which implies (3.6).

Proposition 3.6. On a Kähler foliation $\mathcal{F}$ of codimension $q=2 m$, the following holds: for any basic $r$-form $\phi$,

$$
\begin{aligned}
& {[F, i(\Omega)]=0, \quad\left[\Delta_{B}, i(\Omega)\right]=-\sum_{a=1}^{2 m} i\left(E_{a}\right) i\left(\nabla_{J E_{a}} \kappa_{B}^{\sharp}\right),} \\
& {\left[A_{X}, i(\Omega)\right]=-\sum_{a=1}^{2 m} i\left(E_{a}\right) i\left(\nabla_{J E_{a}} X\right) \quad \forall X \in \Gamma Q,}
\end{aligned}
$$

where $i(\Omega)=-\frac{1}{2} \sum_{a=1}^{2 m} i\left(J E_{a}\right) i\left(E_{a}\right)$.

Proof. Since $\Omega$ is parallel, we have

$$
F(i(\Omega) \phi)=\sum_{a, b=1}^{2 m} \theta^{a} \wedge i\left(E_{b}\right) i(\Omega) R^{\nabla}\left(E_{b}, E_{a}\right) \phi
$$




$$
\begin{aligned}
& =\sum_{a, b=1}^{2 m} \theta^{a} \wedge i(\Omega) i\left(E_{b}\right) R^{\nabla}\left(E_{b}, E_{a}\right) \phi \\
& =i(\Omega) \sum_{a, b=1}^{2 m} \theta^{a} \wedge i\left(E_{b}\right) R^{\nabla}\left(E_{b}, E_{a}\right) \phi+\sum_{a, b=1}^{2 m} i\left(J E_{a}\right) i\left(E_{b}\right) R^{\nabla}\left(E_{b}, E_{a}\right) \phi \\
& =i(\Omega) F(\phi) .
\end{aligned}
$$

The last equality in the above follows from (3.6). On the other hand, by the direct calculation, we have

$$
\left[d_{B}, i(\Omega)\right]=-\sum_{a=1}^{2 m} i\left(E_{a}\right) \nabla_{J E_{a}}, \quad\left[\delta_{B}, i(\Omega)\right]=0 .
$$

From (3.9), the other equations are proved.

An infinitesimal automorphism $Y$ gives rise to a transversally holomorphic field $s=\pi(Y)$ if and only if

$$
\theta(Y) J=0
$$

equivalently,

$$
\nabla_{J Z} s=J \nabla_{Z} s \text { for all } Z \in \Gamma L^{\perp} \text {. }
$$

Hence we have the following corollary.

Corollary 3.7. On a Kähler foliation $\mathcal{F}$, if $\kappa_{B}^{\sharp}$ is transversally holomorphic, then

$$
\left[\Delta_{B}, i(\Omega)\right]=\left[A_{\kappa_{B}^{\sharp}}, i(\Omega)\right]=\delta_{T} i\left(J \kappa_{B}^{\sharp}\right)+i\left(J \kappa_{B}^{\sharp}\right) \delta_{T} .
$$

Proof. The first equality is trivial. For any $r$-form $\phi$, we have

$$
\delta_{T} i\left(J \kappa_{B}^{\sharp}\right) \phi=-\sum_{a=1}^{q} i\left(E_{a}\right) i\left(\nabla_{E_{a}} J \kappa_{B}^{\sharp}\right) \phi-\sum_{a=1}^{q} i\left(E_{a}\right) i\left(J \kappa_{B}^{\sharp}\right) \nabla_{E_{a}} \phi .
$$

Since $\kappa_{B}^{\sharp}$ is transversally holomorphic, $\nabla_{X} J \kappa^{\sharp}=\nabla_{J X} \kappa^{\sharp}$ for any $X \in \Gamma Q$. Hence

$$
\delta_{T} i\left(J \kappa_{B}^{\sharp}\right) \phi=\left[A_{\kappa_{B}^{\sharp}}, i(\Omega)\right] \phi+\sum_{a} i\left(J \kappa_{B}^{\sharp}\right) i\left(E_{a}\right) \nabla_{E_{a}} \phi,
$$

which proves (3.12).

Proposition 3.8. On a Kähler foliation, if $\phi$ is a transverse Killing $r$-form $(r \geq 2)$, then $i(\Omega) \phi$ is a transverse Killing $(r-2)$-form.

Proof. Recall [5] that a basic form $\phi$ is a transverse Killing form if and only if, for any $X \in \Gamma Q$,

$$
i(X) \nabla_{X} \phi=0 .
$$

Since $\Omega$ is parallel, it is trivial that $i(X) \nabla_{X} i(\Omega) \phi=0$ for any $X \in \Gamma Q$. Hence $i(\Omega) \phi$ is a transverse Killing form. 
Theorem 3.9. Let $\left(M, g_{M}, \mathcal{F}, J\right)$ be a compact Riemannian manifold with a Kähler foliation of codimension $q=2 m$ and a bundle-like metric $g_{M}$. Assume that $\kappa_{B}^{\sharp}$ is transversally holomorphic. If $\phi$ is a transverse Killing $r$-form $(r \geq$ $2)$, then $i(\Omega) \phi$ is parallel transverse Killing $(r-2)$-form.

Proof. Let $\phi$ be a transverse Killing $r$-form. By Proposition $3.8, i(\Omega) \phi$ is also a transverse Killing form. Hence, by (3.5), we have

$$
\Delta_{B} i(\Omega) \phi=\frac{r-1}{r-2} F(i(\Omega) \phi)+\theta\left(\kappa_{B}^{\sharp}\right) i(\Omega) \phi .
$$

On the other hand, since $\kappa_{B}^{\sharp}$ is transversally holomorphic and $\delta_{T} \phi=0$, we have from (3.8) and (3.12)

$$
\begin{aligned}
& \Delta_{B} i(\Omega) \phi=i(\Omega) \Delta_{B} \phi+\delta_{T} i\left(J \kappa_{B}^{\sharp}\right) \phi, \\
& i(\Omega) \theta\left(\kappa_{B}^{\sharp}\right) \phi+\delta_{T} i\left(J \kappa_{B}^{\sharp}\right) \phi=\theta\left(\kappa_{B}^{\sharp}\right) i(\Omega) \phi .
\end{aligned}
$$

From (3.7), (3.16) and (3.17), we have

$$
\Delta_{B} i(\Omega) \phi=\frac{r+1}{r} F(i(\Omega) \phi)+\theta\left(\kappa_{B}^{\sharp}\right) i(\Omega) \phi .
$$

From (3.15) and (3.18), we have

$$
F(i(\Omega) \phi)=0, \quad \Delta_{B} i(\Omega) \phi=\theta\left(\kappa_{B}^{\sharp}\right) i(\Omega) \phi .
$$

Hence the generalized Weitzenböck formula (2.12) and (3.19) yield

$$
\Delta_{B}|i(\Omega) \phi|^{2}-\kappa_{B}^{\sharp}\left(|i(\Omega) \phi|^{2}\right)=-2\left|\nabla_{\mathrm{tr}} i(\Omega) \phi\right|^{2} .
$$

Hence $\left(\Delta_{B}-\kappa_{B}^{\sharp}\right)|i(\Omega) \phi|^{2} \leq 0$. By Lemma $2.1,|i(\Omega) \phi|$ is constant. Again, from (3.20), we have

$$
\nabla_{\mathrm{tr}} i(\Omega) \phi=0
$$

which implies that $i(\Omega) \phi$ is parallel.

We define the operators $R_{ \pm}^{\nabla}(X): \wedge^{r} Q^{*} \rightarrow \wedge^{r \pm 1} Q^{*}$ for any $X \in T M$ as

$$
\begin{aligned}
& R_{+}^{\nabla}(X) \phi=\sum_{a=1}^{2 m} \theta^{a} \wedge R^{\nabla}\left(X, E_{a}\right) \phi, \\
& R_{-}^{\nabla}(X) \phi=\sum_{a=1}^{2 m} i\left(E_{a}\right) R^{\nabla}\left(X, E_{a}\right) \phi,
\end{aligned}
$$

where $\theta^{a}$ is a $g_{Q}$-dual 1 -form to $E_{a}$. Trivially, since $i(X) R^{\nabla}=0$ [18] for any $X \in \Gamma L$, if $Y \in V(\mathcal{F})$, then the operators $R_{ \pm}^{\nabla}(Y)$ preserves the basic forms.

Proposition 3.10. Let $\phi \in \Omega_{B}^{r}(\mathcal{F})$ be a transverse Killing $r$-form. Then for all $X \in \Gamma Q$,

$$
\nabla_{X} d_{B} \phi=\frac{r+1}{r} R_{+}^{\nabla}(X) \phi
$$


Proof. Let $\phi$ be a transverse Killing $r$-form. By (3.4), we have

$$
\nabla_{X} \nabla_{Y} \phi=\frac{1}{r+1} i\left(\nabla_{X} Y\right) d_{B} \phi+\frac{1}{r+1} i(Y) \nabla_{X} d_{B} \phi
$$

Hence we have

$$
\nabla_{X, Y}^{2} \phi=\frac{1}{r+1} i(Y) \nabla_{X} d_{B} \phi
$$

So we get

$$
R^{\nabla}(X, Y) \phi=\frac{1}{r+1}\left\{i(Y) \nabla_{X} d_{B} \phi-i(X) \nabla_{Y} d_{B} \phi\right\} .
$$

Since $\sum_{a=1}^{2 m} \theta^{a} \wedge i\left(E_{a}\right) \phi=r \phi$ for any basic $r$-form $\phi$, we have

$$
\begin{aligned}
R_{+}^{\nabla}(X) \phi & =\frac{1}{r+1} \sum_{a=1}^{2 m} \theta^{a} \wedge\left\{i\left(E_{a}\right) \nabla_{X} d_{B} \phi-i(X) \nabla_{E_{a}} d_{B} \phi\right\} \\
& =\nabla_{X} d_{B} \phi-\frac{1}{r+1} \nabla_{X} d_{B} \phi \\
& =\frac{r}{r+1} \nabla_{X} d_{B} \phi
\end{aligned}
$$

which proves (3.24).

Theorem 3.11. Let $\left(M, g_{M}, \mathcal{F}, J\right)$ be a closed, connected Riemannian manifold with a Kähler foliation of codimension $q=2 m$ and a bundle-like metric $g_{M}$. Assume that $\kappa_{B}^{\sharp}$ is transversally holomorphic. Then any transverse Killing $r$-form $(2 \leq r \leq q)$ is parallel.

Proof. Let $\phi$ be a transverse Killing $r$-form. From Theorem $3.9, i(\Omega) \phi$ is parallel. From (3.24) and (3.26), we have

$$
i(\Omega) i(Y) R_{+}^{\nabla}(X) \phi=r \nabla_{X, Y}^{2} i(\Omega) \phi=0
$$

for any $X, Y \in Q$. Since $Y$ is an arbitrary vector field, we have

$$
i(\Omega) R_{+}^{\nabla}(X) \phi=0 .
$$

By a direct calculation, we have

$$
\left[R_{+}^{\nabla}(X), i(\Omega)\right]=R_{-}^{\nabla}(X) .
$$

From (3.28) and (3.29), we have

$$
R_{-}^{\nabla}(X) \phi=0, \quad \forall X,
$$

which implies that

$$
F(\phi)=0 \text {. }
$$

Since $\phi$ is a transverse Killing form, from (3.5)

$$
\Delta_{B} \phi=\theta\left(\kappa_{B}^{\sharp}\right) \phi .
$$


Hence, by the generalized Weitzenböck formula (2.12) and (3.32), we have

$$
\Delta_{B}|\phi|^{2}-\kappa_{B}^{\sharp}\left(|\phi|^{2}\right)=-\left|\nabla_{\mathrm{tr}} \phi\right|^{2} .
$$

Hence, by Lemma 2.1 (the maximum principle), $\phi$ is parallel.

Acknowledgements. This work was supported by the Research grant of Jeju National University in 2009.

\section{References}

[1] J. A. Alvarez López, The basic component of the mean curvature of Riemannian foliations, Ann. Global Anal. Geom. 10 (1992), no. 2, 179-194.

[2] S. D. Jung, Eigenvalue estimates for the basic Dirac operator on a Riemannian foliation admitting a basic harmonic 1-form, J. Geom. Phys. 57 (2007), no. 4, 1239-1246.

[3] M. J. Jung and S. D. Jung, Riemannian foliations admitting transversal conformal fields, Geom. Dedicata 133 (2008), 155-168.

[4] S. D. Jung, K. R. Lee, and K. Richardson, Generalized Obata theorem and its applications on foliations, J. Math. Anal. Appl. 376 (2011), no. 1, 129-135.

[5] S. D. Jung and K. Richardson, Transverse conformal Killing forms and a Gallot-Meyer Theorem for foliations, Math. Z. 270 (2012), 337-350.

[6] F. W. Kamber and Ph. Tondeur, Harmonic foliations, Proc. National Science Foundation Conference on Harmonic Maps, 87-121, Tulance, Dec. 1980, Lecture Notes in Math. 949, Springer-Verlag, New York, 1982.

[7] _ Infinitesimal automorphisms and second variation of the energy for harmonic foliations, Tôhoku Math. J. 34 (1982), no. 2, 525-538.

[8] , De Rham-Hodge theory for Riemannian foliations, Math. Ann. 277 (1987), no. $3,415-431$.

[9] T. Kashiwada and S. Tachibana, On the integrability of Killing-Yano's equation, J. Math. Soc. Japan 21 (1969), 259-265.

[10] M. Min-Oo, E. A. Ruh, and Ph. Tondeur, Vanishing theorems for the basic cohomology of Riemannian foliations, J. Reine Angew. Math. 415 (1991), 167-174.

[11] A. Moroianu and U. Semmelmann, Twistor forms on Kähler manifolds, Ann. Sc. Norm. Super. Pisa Cl. Sci. (5) 2 (2003), no. 4, 823-845.

[12] S. Nishikawa, Ph. Tondeur, Transversal infinitesimal automorphisms for harmonic Kähler foliations, Tôhoku Math. J. 40 (1988), no. 4, 599-611.

[13] J. S. Pak and S. Yorozu, Transverse fields on foliated Riemannian manifolds, J. Korean Math. Soc. 25 (1988), no. 1, 83-92.

[14] E. Park and K. Richardson, The basic Laplacian of a Riemannian foliation, Amer. J. Math. 118 (1996), no. 6, 1249-1275.

[15] U. Semmelmann, Conformal Killing forms on Riemannian manifolds, Math. Z. 245 (2003), no. 3, 503-527.

[16] S. Tachibana, On conformal Killing tensor in a Riemannian space, Tôhoku Math. J. 21 (1969), 56-64.

[17] _ On Killing tensors in Riemannian manifolds of positive curvature operator, Tôhoku Math. J. 28 (1976), 177-184.

[18] Ph. Tondeur, Foliations on Riemannian Manifolds, Springer-Verlag, New-York, 1988.

[19] _ Geometry of Foliations, Birkhäuser-Verlag, Basel; Boston; Berlin, 1997.

[20] K. Yano, Some remarks on tensor fields and curvature, Ann. of Math. 55 (1952), 328347. 
SeOung Dal Jung

Department of Mathematics and Research Institute for Basic Sciences

JeJu National University

JEJU 690-756, KoREA

E-mail address: sdjung@jejunu.ac.kr

Min Joo Jung

Department of Mathematics

Jeju National University

JEJU 690-756, KOREA

E-mail address: nadlehyuk@jejunu.ac.kr 